\title{
Adicción a las redes sociales y habilidades sociales en estudiantes de una institución educativa privada
}

\section{Addiction to Social Networks and Social Skills in Students from a Private Educational Institution}

\author{
Julio A. Domínguez-Vergara* \& Jessenia Ybañez-Carranza \\ Universidad César Vallejo, Trujillo, Perú.
}

Recibido: $18-04-16$

Aprobado: 22-08-16

*Correspondencia

Email: jdominguezv@ucv.edu.pe

\section{Citar como:}

Domínguez, J., \& Ybañez, J. (2016). Adicción a las redes sociales y habilidades sociales en estudiantes de una institución educativa privada. Propósitos y Representaciones, 4(2), 181 - 230. Doi: http://dx.doi.org/10.20511/ pyr2016.v4n2.122

(C) Universidad San Ignacio de Loyola, Vicerrectorado de Investigación y Desarrollo, 2016. (๕) BY.NC-ND Este artículo se distribuye bajo licencia CC BY-NC-ND 4.0 Internacional (http://creativecommons.org/licenses/by-nc-nd/4.0/). 


\section{Resumen}

El presente trabajo de investigación tiene como propósito determinar la relación que existe entre la adicción a las redes sociales y las habilidades sociales en estudiantes de una institución educativa privada. Se realizó un estudio descriptivo correlacional, donde la muestra estuvo representada por 205 estudiantes del 1 ero al 5to grado de secundaria. Se utilizaron dos instrumentos: Lista de Chequeo de Habilidades Sociales - Goldstein y Escala de Actitudes hacia las redes sociales. Esta fue adaptada y validada para la población. Se concluye que la adicción a las redes sociales del Internet tiene una relación significativa $(\mathrm{p}<.01)$ con las habilidades sociales, lo que indica que, a mayor adicción a las redes sociales, se demuestra un bajo nivel de las habilidades sociales en los adolescentes.

Palabras clave: Adicción a las redes sociales, habilidades sociales, estudiantes.

\section{Summary}

This research aims to determine the relationship between addiction to social networks and social skills in students of a private educational centre. A correlational descriptive study where the sample was represented by 205 students from 1st to 5th grade of junior high school was conducted. Two instruments were used: "Goldstein Social Skills Checklist" and "Scale of Attitudes toward Social Networks", this was adapted and validated for the population. It is concluded that addiction to social networks of the Internet has a significant relationship $(\mathrm{p}<.01)$ with social skills, indicating that the greater the addiction to social networks is, a low level of social skills in adolescents is perceived.

Keywords: Addiction to social networks, social skills, students. 


\section{Introducción}

En la actualidad se experimenta una era donde las nuevas tecnologías toman mucho protagonismo como elemento principal de avance y progreso; esto sea en los diferentes ámbitos existentes (personal, familiar, social y empresarial). El impacto de estos instrumentos digitales ha proporcionado grandes beneficios para dar paso a un cambio rotundo en el funcionamiento social de la comunidad. Las nuevas tecnologías se han convertido en un método para poder almacenar y transmitir información de manera continua y permanente, repercutiendo ello en la vida cotidiana, las actividades recreativas y el trabajo en un mundo donde las personas necesitan comunicar. Esta nueva forma de comunicación ha abierto un gran campo de experiencia y aprendizaje, siendo en la actualidad una herramienta indispensable en la vida de las personas. Sin embargo, su inadecuada utilidad ha puesto en riesgo algunas formas de comunicación habitual y de entretenimiento.

Estos recursos tecnológicos han generado tanta relevancia dentro de los estilos de vida de cada persona que poco a poco han ido deteriorando las relaciones familiares, sociales y laborales. Los principales consumidores de las nuevas tecnologías son los adolescentes, generación que ha transformado la comunicación habitual en un método de interacción digital; asimismo, ellos transmiten esta cultura de tecnología sobre otras personas, enseñando incluso a los adultos, quienes adolecen de falta de capacidad para poder adaptarse al continuo cambio de la tecnología. Por ello, los adolescentes se constituyen como el sector que más fácil se adapta a las nuevas tecnologías.

El excesivo uso de las nuevas tecnologías se ve reflejado en una encuesta realizada por la Asociación para la Investigación de los Medios de Comunicación (AIMC, 2001), en la que un 25\% de las 43942 personas encuestadas reconoce que ha disminuido el tiempo dedicado a dormir; un $13.8 \%$, el tiempo dedicado al estudio; un $10.1 \%$, el tiempo dedicado a salir con los amigos, y un $6.1 \%$ dice que el uso de Internet ha disminuido el tiempo laboral. El carácter marcadamente lúdico del uso de Internet 
se pone de manifiesto aún más al señalar este informe que el $65 \%$ de los encuestados reconoce que las conexiones a la red han hecho disminuir el tiempo dedicado a ver la televisión, y un 58.3\% afirma que la red ha llenado un tiempo vacío dedicado al ocio. Otro dato significativo proporcionado por este informe es que solo un $9.5 \%$ de los usuarios encuestados utiliza la red con finalidades principalmente académicas. Del resto, un 39.1\% la utiliza con fines profesionales o laborales, y un $50.2 \%$ lo hace con fines personales.

Las nuevas tecnologías se han instaurado de la mejor manera entre los adolescentes, destronando a los medios de comunicación, como la televisión, la radio, el teléfono y el cine, que son útiles y que resultan atractivos para una gran parte de la población. Sin embargo, las nuevas tecnologías digitales de entretenimiento parecen haber llevado el concepto de placer a un nuevo nivel y con una eficiencia, disponibilidad y facilidad de acceso nunca vistas antes en la sociedad moderna (Echeburúa \& Requenses, 2012).

Yang (2001) aporta datos en la misma dirección. Este autor concluye que, entre los múltiples usos que se pueden hacer del ordenador, solo un $8.5 \%$ de los sujetos estudiados lo utiliza con fines educativos o relacionados con actividades escolares. La mayoría de los usuarios utiliza el ordenador como una fuente de entretenimiento y ocio, sobre todo a través de los juegos y las redes sociales.

En esta última década, las redes sociales han puesto de manifiesto la capacidad inagotable de innovación de las industrias de la información y el entretenimiento. La difusión acelerada de esta nueva manera de comunicarse (o de identificar personas con las cuales se quiere entablar comunicación) y de crear contenido individualizado hace plantearse preguntas tales como hasta qué punto se está presenciando un cambio radical en el uso de medios de comunicación.

El aumento de popularidad de las redes sociales ha trascurrido en paralelo al aumento en los niveles de intercambio de contenidos a través de la red. Esto ha hecho del Internet un medio más social que permite comunicar, 
entretener y compartir. Los usuarios han pasado de una etapa en la que eran considerados meros consumidores de contenidos creados por terceros usuarios con ciertos conocimientos de programación, a una etapa en la que los contenidos son producidos por los propios usuarios equipados con un ordenador, conexión y conocimientos básicos en el uso de Internet.

Choliz y Marco (2012) consideran que las redes sociales son las herramientas más utilizadas por jóvenes y adolescentes, ya que tienen la posibilidad de ampliar el círculo de amigos, así como de conectarse de manera sencilla con otras personas para compartir experiencias o información. Estas características han hecho de las redes sociales una herramienta indispensable para el uso de la comunicación, donde los usuarios puedan disponer de su propia página online (home page), crear un perfil para colgar sus fotos, elaborar comentarios sobre sí mismos y sus contactos, hablar de sus gustos e intereses y compartir experiencias e información (Carbonell, 2014). El impacto en los adolescentes por estas características de las redes sociales ha generado un elevado auge en pocos años de existencia.

El acceso y la multifuncionalidad que ofrecen las redes sociales son elementos atractivos que encuentran los adolescentes para conectarse; es decir, los adolescentes pueden tener libre acceso a todo tipo de contenido mediante el uso de las redes sociales y, paralelamente, pueden realizar tareas, ya que pueden abrir más de dos pestañas mediante las páginas web o pueden escuchar música y hacer la tarea al mismo tiempo. Ello genera en el adolescente sensaciones de placer, cambios conductuales y emocionales, evidenciándose una adicción psicológica (Echeburúa \& Requenses, 2012).

Las consecuencias que experimentan los adolescentes se reflejan en el sueño alterado, ya que prefieren estar conectados durante las noches, disminuyendo las horas de sueño; otro aspecto es el patrón de hambre alterado, que consiste en comer rápido $\mathrm{y}$ desordenadamente para poder conectarse inmediatamente; asimismo, descuido de la higiene personal y cambio de estilo de ocio, donde existe un desinterés absoluto por el deporte $\mathrm{u}$ otras actividades para estar conectado a las redes sociales. También se 
mencionan consecuencias que parten desde la irritabilidad, estado de humor oscilante y bajo rendimiento académico (Matalí \& Alda, 2008).

En el contexto social peruano, el Instituto Nacional de Estadística e Informática (2011), a través de una Encuesta Nacional de Hogares, observó que un $43.3 \%$ de mujeres y un $38.6 \%$ de varones entre 11 y 20 años de edad usan Internet. Asimismo, Cruzado, Matos y Kendall (2006) refieren que el Perú es el segundo país latinoamericano en cuanto a tasa de penetración de Internet (11,5 usuarios/100 habitantes), y la Organización Mundial de la Salud (2008) señala que una de cada cuatro personas sufre trastornos de conducta y problemas en sus relaciones interpersonales relacionados con las nuevas adicciones.

La provincia de Trujillo se considera una ciudad muy violenta, impulsiva e insegura. Este síntoma de violencia se debe a múltiples factores en la formación de los adolescentes, donde padres irresponsables no asumen un rol funcional sobre su crianza. De tal modo, los adolescentes toman actitudes de riesgo en su afán por conseguir sensaciones de placer. Una de estas causas son las nuevas tecnologías. Estos recursos digitales transportan la atención y conciencia de los adolescentes a estados de bienestar y comodidad, adquiriendo vulnerabilidad a la adicción.

Los adolescentes han sido considerados grupos de riesgo por muchos autores, por las características propias de este momento evolutivo: omnipotencia, tendencia a buscar la causa de sus problemas en el otro, poca experiencia de vida, dificultad en reconocer adicciones sutiles y sensación de normalidad ante conductas de riesgo (Castellana \& Llado, 1999).

El presente trabajo de investigación muestra la relación entre la adicción a las redes sociales y las habilidades sociales, poniendo énfasis en la forma de comunicación que se emplea en el mundo actual por parte de los adolescentes a través del Internet y analizando las dimensiones de las habilidades sociales del mismo adolescente para con su entorno social (escuela y familia). 


\section{Método}

Tipo y diseño de investigación.

La presente investigación es de carácter sustantivo debido a que complementa elementos de investigación aplicada y pura. Asimismo, se explican y describen los hallazgos del estudio desde una perspectiva teórica y su utilidad para la población beneficiaria. El diseño de estudio en el que se enmarca es descriptivo correlacional, ya que se miden dos variables dentro de un mismo grupo de personas (Sánchez \& Reyes, 2006).

\section{Participantes.}

La muestra está conformada por 205 adolescentes de ambos sexos (104 varones y 101 mujeres), entre 12 y 16 años de edad, del 1ero al 5to año de secundaria de una institución educativa particular. Se utilizó un muestreo probabilístico estratificado donde las personas que integran la población tienen la posibilidad de pertenecer a la muestra (acorde con el perfil estipulado). La muestra se subdividió en estratos (diferentes muestras para diferentes grados) (Palella \& Martins, 2006)

\section{Tabla 1.}

Muestra estratificada de los estudiantes de una institución educativa privada.

\begin{tabular}{cccc}
\hline Grado & Varones & Mujeres & Total \\
\hline $1^{\circ}$ & 23 & 21 & 44 \\
$2^{\circ}$ & 19 & 24 & 43 \\
$3^{\circ}$ & 21 & 17 & 39 \\
$4^{\circ}$ & 19 & 20 & 39 \\
$5^{\circ}$ & 21 & 18 & 40 \\
Totales & $\mathbf{1 0 4}$ & $\mathbf{1 0 1}$ & $\mathbf{2 0 5}$ \\
\hline
\end{tabular}

\section{Instrumentos.}

Para la investigación utilizamos los siguientes instrumentos: 
a) Escala de actitudes hacia las redes sociales.

Ficha técnica: Escala de actitudes hacia las redes sociales, creada por Domínguez (2011), de procedencia peruana, administración individual y colectiva. La estimación del tiempo de aplicación oscila entre 10 y 15 minutos, y comprende un ámbito de aplicación en adolescentes de 12 a 16 años de edad.

La elaboración del cuestionario se realizó en una muestra de 100 alumnos entre las edades de 12 y 16 años. Se determinó la validez de contenido mediante la técnica de criterio de jueces ya que, según Kerlinger (1975, como se citó en Alarcón, 2013), la validez de contenido es verificada por jueces expertos, quienes evalúan la representatividad de los indicadores de la conducta que se mide, buscando establecer si representan el universo del contenido de esa conducta y la relevancia de los ítems para medir tales indicadores. Se presentó una tabla de especificaciones a 10 jueces con un total de 51 ítems, de los cuales mediante el coeficiente $\mathrm{V}$ de Aiken alcanzaron valores entre 0.8 y 1.00 , indicando que fueron válidos y adecuados los cambios realizados $(\geq .80$ ) (Merino \& Livia, 2009). Para la validez de constructo se efectuó la correlación ítem test, donde la escala alcanzó valores por encima de 0.2 (Kline, 2006) en la mayoría de ítems, razón por la cual el test mantiene homogeneidad sobre el constructo a medir; sin embargo, 14 ítems no alcanzaron el valor sugerido, siendo estos eliminados y conformándose el instrumento por 37 ítems. La confiabilidad alcanzó una consistencia interna de Alpha Cronbach de 0.95. Asimismo, se halló el promedio de las calificaciones totales por evaluado, hallando un valor de 53; luego, una desviación estándar de 11. Así, mediante la suma del promedio más la desviación estándar, obtuvimos los puntajes altos para las categorías: altamente significativo (37-41) y significativo (42-51), mientras que al restar el promedio con la desviación estándar se obtuvieron los puntajes bajos para las categorías poco significativo (52-64) y no significativo (37-41). 
b) Lista de chequeo de habilidades sociales de Goldstein.

Creada por Goldstein (1980) y adaptada al español por Tomas (1995). La administración del instrumento es de carácter individual y colectivo. La estimación del tiempo de aplicación oscila entre 10 y 15 minutos, y comprende un ámbito de aplicación en adolescentes a partir de los 12 años. Los baremos fueron obtenidos en una muestra de 100 alumnos entre las edades de 12 y 16 años. Según la validez criterial, el instrumento tiene validez ya que presenta diferencias significativas entre los promedios de los niveles alto y bajo en cada área. Por último, de acuerdo con la consistencia interna de Alpha de Cronbach, la lista de chequeo de habilidades sociales es confiable debido a que su valor es de 0.94 .

\section{Procedimiento.}

La aplicación de los instrumentos se realizó en el aula de las clases teóricas habituales, en el día y hora acordados con el coordinador de curso. Las secciones que participaron fueron seleccionadas al azar. Antes de la administración de los instrumentos psicológicos se aclaró a los encuestados sobre los criterios básicos de la selección de la muestra, así como de las condiciones sobre su participación en la investigación. Los participantes, con conocimiento de los fines de estudio y del empleo de la información, firmaron la carta de asentimiento informado y, posteriormente, los alumnos empezaron con el llenado de las pruebas.

\section{Resultados}

En la Tabla 1 se observan los niveles de adicción a las redes sociales del Internet, donde se destaca un nivel medianamente significativo representado por 95 estudiantes, quienes abarcan el $46.3 \%$ de la muestra. 


\section{Tabla 2.}

Distribución de frecuencias y niveles de la adicción a las redes sociales del Internet.

\begin{tabular}{lcc}
\hline Categorías & N & \% \\
\hline Nada significativo & 0 & 0.0 \\
Poco significativo & 57 & 27.8 \\
Medianamente significativo & 95 & 46.3 \\
Significativo & 30 & 14.6 \\
Altamente significativo & 23 & 11.2 \\
Total & 205 & 100.0 \\
\hline
\end{tabular}

En la Tabla 2 se puede observar que los adolescentes se caracterizan por presentar en el grupo I, de Habilidades sociales, un nivel Medio representado por el $40.0 \%$; en el grupo II, de Habilidades sociales avanzadas, un nivel Medio expresado en un $46.8 \%$; en el grupo III, de Habilidades sociales alternativas a la agresión, los adolescentes alcanzan en mayor número un nivel Alto representado en un 38.5\%; en el grupo IV, de Habilidades para la planificación, los adolescentes se ubican más en un nivel Alto expresado en un $37.1 \%$; en el grupo V, de Primeras habilidades sociales, se refleja mayor número de adolescentes en el nivel Alto representado por un $40.0 \%$; por último, en el grupo VI, de Habilidades sociales para hacer frente al estrés, se refleja mayor número de adolescentes en el nivel Medio expresado en un $36.6 \%$.

\section{Tabla 3.}

Distribución de frecuencias y niveles de las habilidades sociales.

\begin{tabular}{llll}
\hline Escala & Nivel & $\mathbf{N}$ & $\mathbf{\%}$ \\
\hline \multirow{3}{*}{ Grupo I } & Bajo & 51 & 24.9 \\
(Habilidades sociales) & Medio & 82 & 40.0 \\
& Alto & 72 & 35.1 \\
& Total & 205 & 100.0 \\
\hline
\end{tabular}




\begin{tabular}{|c|c|c|c|}
\hline \multirow{4}{*}{$\begin{array}{l}\text { Grupo II } \\
\text { (Habilidades sociales } \\
\text { avanzadas) }\end{array}$} & Bajo & 46 & 22.4 \\
\hline & Medio & 96 & 46.8 \\
\hline & Alto & 63 & 30.7 \\
\hline & Total & 205 & 100.0 \\
\hline \multirow{4}{*}{$\begin{array}{l}\text { Grupo III } \\
\text { (Habilidades sociales } \\
\text { alternativas a la agresión) }\end{array}$} & Bajo & 52 & 25.4 \\
\hline & Medio & 74 & 36.1 \\
\hline & Alto & 79 & 38.5 \\
\hline & Total & 205 & 100.0 \\
\hline \multirow{4}{*}{$\begin{array}{l}\text { Grupo IV } \\
\text { (Habilidades de } \\
\text { planificación) }\end{array}$} & Bajo & 60 & 29.3 \\
\hline & Medio & 69 & 33.7 \\
\hline & Alto & 76 & 37.1 \\
\hline & Total & 205 & 100.0 \\
\hline \multirow{4}{*}{$\begin{array}{l}\text { Grupo V } \\
\text { (Primeras habilidades } \\
\text { sociales) }\end{array}$} & Bajo & 64 & 31.2 \\
\hline & Medio & 59 & 28.8 \\
\hline & Alto & 82 & 40.0 \\
\hline & Total & 205 & 100.0 \\
\hline \multirow{4}{*}{$\begin{array}{l}\text { Grupo VI } \\
\text { (Habilidades sociales para } \\
\text { hacer frente al estrés }\end{array}$} & Bajo & 57 & 27.8 \\
\hline & Medio & 75 & 36.6 \\
\hline & Alto & 73 & 35.6 \\
\hline & Total & 205 & 100.0 \\
\hline
\end{tabular}

En la Tabla 3 se muestran correlaciones inversas de grados altos $(\mathrm{p}<.01)$ entre la adicción a las redes sociales y las habilidades sociales, habilidades sociales avanzadas, habilidades sociales alternativas a la agresión, habilidades de planificación, primeras habilidades sociales y habilidades sociales para hacer frente al estrés. 


\section{Tabla 4.}

Correlación entre la adicción a las redes sociales del Internet y las habilidades sociales.

\begin{tabular}{llccc}
\hline \multicolumn{1}{c}{ V1 } & \multicolumn{1}{c}{ V2 } & r & Sig & \\
\hline $\begin{array}{l}\text { Adicción a las redes } \\
\text { sociales }\end{array}$ & Habilidades sociales & -0.696 & 0.000 & $* *$ \\
$\begin{array}{l}\text { Adicción a las redes } \\
\text { sociales }\end{array}$ & Habilidades sociales avanzadas & -0.679 & 0.000 & $* *$ \\
$\begin{array}{l}\text { Adicción a las redes } \\
\text { sociales }\end{array}$ & $\begin{array}{l}\text { Habilidades sociales alternativas a la } \\
\text { agresión }\end{array}$ & -0.566 & 0.000 & $* *$ \\
$\begin{array}{l}\text { Adicción a las redes } \\
\text { sociales }\end{array}$ & Habilidades de planificación & -0.693 & 0.000 & $* *$ \\
$\begin{array}{l}\text { Adicción a las redes } \\
\text { sociales }\end{array}$ & Primeras habilidades sociales & -0.697 & 0.000 & $* *$ \\
$\begin{array}{l}\text { Adicción a las redes } \\
\text { sociales }\end{array}$ & $\begin{array}{l}\text { Habilidades sociales para hacer frente } \\
\text { al estrés }\end{array}$ & -0.708 & 0.000 & $* *$ \\
\hline
\end{tabular}

$* * p<.01$

\section{Discusión}

De acuerdo con los resultados presentados anteriormente, llegamos a las siguientes reflexiones: existe una relación inversa altamente significativa entre la adicción a las redes sociales del Internet y las habilidades sociales en los adolescentes. Lo que indicaría que, a medida que la adicción a las redes sociales se incrementa, el nivel de habilidades sociales decrece y viceversa. Esto obedecería a lo expuesto por Goldberg (1995), quien sostuvo que los factores de riesgo más implicados en esta adicción son: el aburrimiento y carencia de relaciones u objetivos, la falta de habilidades para desenvolverse en el mundo real, la timidez, la búsqueda de sensaciones en personas solitarias y la ausencia de una autoestima adecuada. Asimismo, Jiménez y Pantoja (2007) refieren que un adicto, en general, es una persona que presenta inhabilidad social o menor habilidad para soportar los aumentos de su tensión psíquica. En contraste, los sujetos no adictos son más equilibrados y logran un mayor control de sí mismos cuando están frente a elementos estresantes tanto internos como externos. Es por ello que el joven sin 
amigos tiende a utilizar excesivamente el ordenador, donde la constante lucha contra el aburrimiento se enmarca en la búsqueda de relaciones online (Ceyhan, 2008).

Existe una correlación inversa altamente significativa entre las habilidades sociales avanzadas y la adicción a las redes sociales. Estos resultados guardan relación con lo expuesto por Petrie y Gunn (1998), asegurando que las personas dependientes del Internet suelen ser susceptibles, vigilantes y privadas, orientadas a una tendencia hacia la introversión.

Sin embargo, en contraposición a estos resultados, Balaguer (2003) demostró que el ciberespacio, y particularmente el chat, el messenger y las redes sociales, proveen a los sujetos de un nuevo espacio psicosocial en el cual pueden tener cierta catarsis de la vida posmoderna, así como desplegar aspectos personales y sociales, de forma tal que la historia no guarda antecedentes. Asimismo, permite a millones de jóvenes establecer comunicación entre sí, lo que genera que se llegue a cifras de usuarios tan altas como las alcanzadas por sitios como Myspace, Facebook o Flickr. La discrepancia entre estas posturas se debería posiblemente a que gran parte de la investigación hasta ahora descrita sobre la adicción a las redes sociales presenta dificultades metodológicas referidas a los procedimientos de selección de la muestra a través de voluntarios procedentes de la propia red (Young, 1998), que pueden ser únicamente representativos de un subgrupo específico de internautas.

Existe una correlación inversa altamente significativa entre las habilidades alternativas a la agresión y la adicción a las redes sociales del Internet, lo que indicaría que, a mayor habilidad de autocontrol, defender sus derechos, responder a las bromas, evitar problemas con los demás y no entrar en problemas, menor será la necesidad de pasar más tiempo conectado a su red social. Para corroborar dicho resultado se presenta lo investigado por Echeburrúa (1999), quien sostiene que el medio en el que se desenvuelve la adicción acarrea también una serie de cambios psicológicos negativos, consistentes en alteraciones del humor, ansiedad o impaciencia por la lentitud 
de las conexiones o por no encontrar lo que se busca o a quien se busca, estado de conciencia alterado (total focalización atencional), irritabilidad en caso de interrupción, incapacidad para salirse de la pantalla, etc.

Existe una correlación inversa altamente significativa entre las habilidades de planificación y la adicción a las redes sociales del Internet, lo que indicaría que, a mayor habilidad para tomar decisiones, establecer objetivos, resolver los problemas según su importancia y concentrarse en una determinada tarea, menor será la necesidad de conectarse a la red social. Choliz y Marco (2012) mencionan que la pérdida de control sobre el uso del Internet genera un aumento significativo de conexiones a la red, empleando gran parte del tiempo en actividades relacionadas con las redes sociales o el Internet y dejando de realizar otras actividades. Echeburrúa (1999) afirma también, al respecto, que el adicto se aísla del entorno y no presta atención a otros aspectos de las obligaciones sociales.

Existe una correlación inversa altamente significativa entre las primeras habilidades sociales y la adicción a las redes sociales del Internet, lo que indicaría que, a mayor habilidad para interactuar con los demás como: escuchar, iniciar una conversación y presentar a otras personas, menor será la necesidad de pasar más tiempo conectado a su red social. Esto concordaría con lo expuesto por Hirsh (1985), quien sostuvo que durante la etapa de la adolescencia se comienza a articular un repertorio de identidades sociales, siendo la tarea principal formar nuevas redes sociales que reflejen estas nuevas identidades, por lo que una conducta adictiva hacia las redes sociales del Internet afectaría dicha tarea. En oposición a esta postura, para Echeburrúa (1999), el uso de las redes sociales de manera adecuada podría permitir la satisfacción de dos tipos de necesidades básicas para los adolescentes: por un lado, la estimulación solitaria (búsqueda de información de imágenes o incluso de sonidos, juegos solitarios, etc.) y, por el otro, la búsqueda de interacción social (conversaciones, comentarios, etc.).

Existe una correlación inversa altamente significativa entre las habilidades sociales para hacer frente al estrés y la adicción a las redes sociales del 
Internet, lo que indicaría que, a mayor habilidad para responder al fracaso, responder persuasiones, enfrentarse a mensajes contradictorios, prepararse para conversaciones difíciles y hacer frente a presiones de grupo, menor será la necesidad de pasar más tiempo conectado a su red social. Esto obedecería a lo investigado por Arón, Sarquis y Machuca (1990), quienes sostienen que la red social influye en la forma en que los adolescentes enfrentan al mundo, su perspectiva del mundo y de sí mismos. Además, Young (1998) manifestó que los problemas surgidos de la dependencia trascienden el ámbito de lo intrapersonal. Desde un punto de vista sistémico, los efectos negativos de la adicción se expresan en el ámbito familiar y el trato de pares.

Las habilidades sociales relacionadas con los sentimientos y la adicción a las redes sociales del Internet son independientes. Esto se fundamentaría por lo expuesto por Turkle (1997): muchos usuarios reportan sentirse más capaces de mostrarse "auténticos" ya que varios de los juegos que se dan en la interacción cara a cara no están presentes en la interacción online. Esta es una de las paradojas de este nuevo mundo altamente tecnificado. Las relaciones virtuales permiten ser más "uno mismo". Por eso, los más jóvenes se animan a declararse a sus novias, y los adultos, a flirtear y mostrar sus facetas más oscuras. Es por esto también que pasan tantas horas en dichos entornos virtuales. Moody (2001 como se citó en Carbonell, 2014) refiere que las personas con sobreutilización de las redes sociales presentan una elevada soledad emocional con pocas relaciones significativas, siendo muy difícil confiar y compartir intimidades. Finalmente, podríamos hablar de aquellos a quienes con cierta tendencia a la "introversión" les asaltan pensamientos negativos sobre su capacidad para interaccionar con otros, incomodándoles en las relaciones sociales. Esta situación incómoda propiciaría buscar relaciones "reforzantes" a través de otros medios con los que logran "evitar" la presencia física o exposición directa en situaciones sociales; se puede mantener el anonimato o elaborar una personalidad "ficticia" o, también, abandonar la "relación virtual" sin consecuencias negativas directamente perceptibles (Young, 1998). Esto demostraría que la habilidad para comprender a los demás, expresar afecto y autocompensarse no resultaría 
perjudicada sustancialmente por la presencia de conductas adictivas hacia las redes sociales del Internet.

Con respecto a la adicción a las redes sociales del Internet, se encontró que, por cada 10 alumnos, cinco presentan tendencias a desarrollar conductas adictivas hacia estas, lo que se reflejaría en los estudios realizados por Greenfield (1999), donde se demostraría que esta nueva adicción es más peligrosa entre los más jóvenes, ya que seis de cada 10 niños y adolescentes corren el riesgo de volverse tecnoadictos. Asimismo, según un informe que recopila datos sobre Internet y las redes sociales, provisto por la agencia de medios Mindshare, el 45\% de los adolescentes argentinos navega en Internet los siete días de la semana; casi un $15 \%$ permanece conectado más de tres horas por día, un $10 \%$ navega entre dos y tres horas, mientras que casi el $35 \%$ (el segmento mayoritario) lo hace entre una y dos horas diarias. Sin embargo, y contrariamente a estos estudios, parece haber quedado claro que el tiempo no es una razón válida para el diagnóstico. Muchos individuos, por razones de trabajo o estudios, deben pasar muchas horas conectados, delante de la computadora, pero eso no les convierte automáticamente en adictos (Lamb \& Davidson, 2002).

Finalmente, y basados en los resultados globales, inferimos que cualquier comportamiento normal placentero es susceptible de convertirse en una conducta adictiva si la persona pierde el control cuando desarrolla una actividad determinada, continúa con ella a pesar de las consecuencias adversas, muestra una dependencia cada vez mayor de esa conducta que está precipitada por un sentimiento que puede oscilar desde un deseo moderado hasta una obsesión intensa-, sufre síndrome de abstinencia si no puede practicarla y, por último, pierde interés por otro tipo de conductas que previamente le resultaban satisfactorias. Más aún que en esta nueva cultura digital se presentan posibilidades de expresión, agrupamientos y búsqueda de satisfacciones libidinales que nuestra cultura occidental parecía ya no brindar.

Sin embargo, aun cuando las personas hagan un uso inadecuado y contraproducente de las redes sociales del Internet, resulta prematuro 
referirnos a un trastorno de adicción. Navegar por la red puede ser, en sentido estricto, una pauta de comportamiento adictivo donde aparecen síntomas de abstinencia, pérdida de control, abuso y obsesión, pero no suele caracterizarse por la adopción de conductas tan autodestructivas como en el caso del resto de las adicciones (Echeburrúa, 1999). En ese sentido, la incorporación de la tecnología en las escuelas parte de la búsqueda auténtica en la renovación metodológica y transformación educativa, donde el nuevo marco de competencia exige cambios profundos en los procesos de enseñanza-aprendizaje (Carbonell, 2014).

\section{Limitaciones y sugerencias}

Los hallazgos obtenidos se limitan a este grupo de estudiantes. Sería conveniente ampliar la muestra en otros grupos de aplicación para extender la generalización de los resultados. Asimismo, una recomendación para futuros estudios es conocer el comportamiento de las variables en otras poblaciones con características diferentes, de modo que se busque confirmar los hallazgos obtenidos.

\section{Referencias}

Alarcón, R. (2013). Métodos y diseños de Investigación del Comportamiento. Lima: Editorial Universitaria.

Arón, A., Sarquis, C., \& Machuca, A. (1990). Consideración de las redes sociales en las intervenciones terapéuticas. Una estrategia de prevención. Ponencia presentada en el Noveno Congreso de Psicología Clínica, Chile.

Asociación para la Investigación de Medios de Comunicación [AIMC]. (2001). Cuarta encuesta AIMC a usuarios de Internet. Recuperado de: http://www.aimc.es.

Balaguer, R. (2003). Internet: un nuevo espacio psicosocial. Montevideo: Trilce.

Carbonell, X. (2014). Adicciones tecnológicas: Qué son y cómo tratarlas. Madrid: Síntesis. http://dx.doi.org/10.20882/adicciones.10 
Castellana, M., \& Llado, M. (1999). Adolescencia y juventud: prevención y percepción del riesgo al consumo. Revista española de Drogodependencia, $2,118-131$.

Chóliz, M., \& Marco, C. (2012). Adicción a internet y redes sociales. Madrid: Alianza Editorial.

Ceyhan, A. (2008). University Students' Problematic Internet Use and Communication Skills according to the Internet Use Purposes. Educational Sciences: Theory \& Practice. 11(1), 69-77.

Cruzado, L., Matos, L., \& Kendall, R. (2006). Adicción a internet: Perfil clínico y epidemiológico de pacientes hospitalizados en un instituto nacional de salud mental. Revista Médica Herediana. 17(4), 196-205. http://dx.doi.org/10.20453/rmh.v17i4.879

Domínguez, J. (2011). Propiedades psicométricas de la Escala de Actitudes hacia las redes sociales (Tesina de licenciatura). Universidad César Vallejo, Trujillo.

Echeburúa, E. (1999). ¿Adicciones sin drogas? Las nuevas adicciones. Bilbao: Desclée de Brouwer.

Echeburúa, E., \& Requenses, A. (2012). Adicción a las redes sociales y nuevas tecnologías en niños y adolescentes. Madrid: Pirámide.

Goldberg, J. (1995). Internet addiction disorder: Diagnostic criteria. Recuperado de: www-usr.rider.edu/ suler/psycyber/supportgp.html.

Goldstein, A. (1980). Lista de Chequeo de Habilidades Sociales (LCHS). Madrid: Projective Way.

Greenfield, D. (1999). Psychological characteristics of compulsive Internet use: a preliminary analysis. Cyberpshychology and behaviour, 2, 403412. http://dx.doi.org/10.1089/cpb.1999.2.403

Hirsch, B. (1985). Social network and the ecology of human development. Social support: theory research and aplications, 117-132.

Instituto Nacional de Estadística e Informática [INEI]. (25 de septiembre de 2011). Las tecnologías de información y comunicación en los hogares. Informe técnico octubre-noviembre-diciembre. Recuperado de https:// www.inei.gob.pe/web/Biblioinei/BoletinFlotante.asp?file=13036. $\operatorname{pdf} \% 253 \mathrm{E}$ 
Jimenez, L., \& Pantoja, A. (2007). Autoestima y relaciones interpersonales en sujetos adictos a Internet. Psicología-Segunda Época, 26(1), 78-89.

Kline, R. B. (2006). Principios y práctica de modelado de la ecuación estructural. New York: Guildford Press.

Lamb, R., \& Davidson, E. (2002). Social scientist: Managing identity in sociotechnical networks. Proceedings of the Hawai's International Conference on System Sciences. http://dx.doi.org/10.1109/HICSS.2002.994034

Matali, J., \& Alda, J. (2008). Adolescentes y nuevas tecnologías. Innovación o adicción. Barcelona: Edebe.

Merino, C., \& Livia, J. (2009). Intervalos de confianza asimétricos para el índice de la validez de contenido: Un programa Visual Basic para la $\mathrm{V}$ de Aiken. Anales de psicología, 25, 169-171.

Organización Mundial de la Salud (2008). Descripciones clínicas y pautas para el diagnóstico. Madrid: Meditor.

Palella, S., \& Martins, F. (2006). Metodología de la Investigación Cuantitativa. Caracas: Fondo Editorial de la Universidad Pedagógica Libertador (FEDEUPEL).

Petrie, H., \& Gunn, D. (1998). Internet "addiction": the effects of sex, age, depression and introversion. British Psychological Society London Conference.

Sánchez, H., \& Reyes, C. (2006). Metodología y diseños en la investigación científica. Lima: Visión Universitaria.

Tomas, A. (1995). Conducta Tipo A y habilidades sociales en estudiantes del primer al quinto año de Psicología de una Universidad Nacional de Lima (Tesis de licenciatura). Universidad Federico Villarreal, Lima.

Turkle, S. (1997). La vida en pantalla: La identidad en la era de Internet. Barcelona: Paidós.

Yang, C. (2001). Sociopsychiatric characteristics of adolescents who use computers to excess. Acta Psychiatrica Scandinavica, 104, 217-222. http://dx.doi.org/10.1034/j.1600-0447.2001.00197.x

Young, K. (1998). Internet addiction: The emergence of a new clinical disorder. Cyberpsychology and behavior, 1(3), 237-244. http://dx.doi. org/10.1089/cpb.1998.1.237 


\section{Anexo A}

\section{Escala de actitudes hacia las redes sociales del Internet.}

\section{A continuación encontrarás una serie de afirmaciones en relación con las redes sociales en Internet como: Twiter, Facebook, MySpace, etc. Marca con una $\mathbf{X}$ la respuesta que más se adecúe a tus comportamientos, pensamientos y sentimientos. No existen respuestas buenas ni malas. No dejes de responder ninguna pregunta y trata de ser lo más sincero posible.}

\begin{tabular}{|c|c|c|c|}
\hline & Siempre & A veces & Nunca \\
\hline $\begin{array}{l}\text { Siento que necesito más tiempo para revisar mis redes } \\
\text { sociales. }\end{array}$ & & & \\
\hline $\begin{array}{l}\text { Finjo estar concentrado en asuntos importantes, cuando } \\
\text { en realidad estoy pensando si en mis redes sociales ha } \\
\text { habido cambios. }\end{array}$ & & & \\
\hline El pensar en las redes sociales distrae mis pensamientos. & & & \\
\hline $\begin{array}{l}\text { Me siento intranquilo si dejo de revisar mis redes } \\
\text { sociales varios días seguidos. }\end{array}$ & & & \\
\hline $\begin{array}{l}\text { Tengo dificultades para controlar mis impulsos de } \\
\text { conectarme a las redes sociales. }\end{array}$ & & & \\
\hline $\begin{array}{l}\text { Me impaciento cuando no estoy revisando las } \\
\text { actualizaciones en mis redes sociales. }\end{array}$ & & & \\
\hline Sueño que conozco gente a través de mis redes sociales. & & & \\
\hline $\begin{array}{l}\text { Tengo sueños confusos entre comentarios reales y lo que } \\
\text { se publica en mis redes sociales. }\end{array}$ & & & \\
\hline $\begin{array}{l}\text { Me siento tranquilo cuando estoy revisando mis redes } \\
\text { sociales. }\end{array}$ & & & \\
\hline $\begin{array}{l}\text { Al revisar mis redes sociales, siento que mi angustia } \\
\text { disminuye. }\end{array}$ & & & \\
\hline $\begin{array}{l}\text { Me agrada estar conectado al Internet revisando mis } \\
\text { redes sociales. }\end{array}$ & & & \\
\hline Estar revisando mis redes sociales me satisface. & & & \\
\hline $\begin{array}{l}\text { Me entusiasma el hecho de saber que tengo comentarios } \\
\text { nuevos. }\end{array}$ & & & \\
\hline $\begin{array}{l}\text { Mi frustración disminuye cuando estoy conectado a mis } \\
\text { redes sociales. }\end{array}$ & & & \\
\hline $\begin{array}{l}\text { Me molesto cuando alguien me interrumpe mientras } \\
\text { reviso mis redes sociales. }\end{array}$ & & & \\
\hline $\begin{array}{l}\text { Prefiero revisar mis redes sociales antes que hacer mis } \\
\text { labores en casa. }\end{array}$ & & & \\
\hline
\end{tabular}




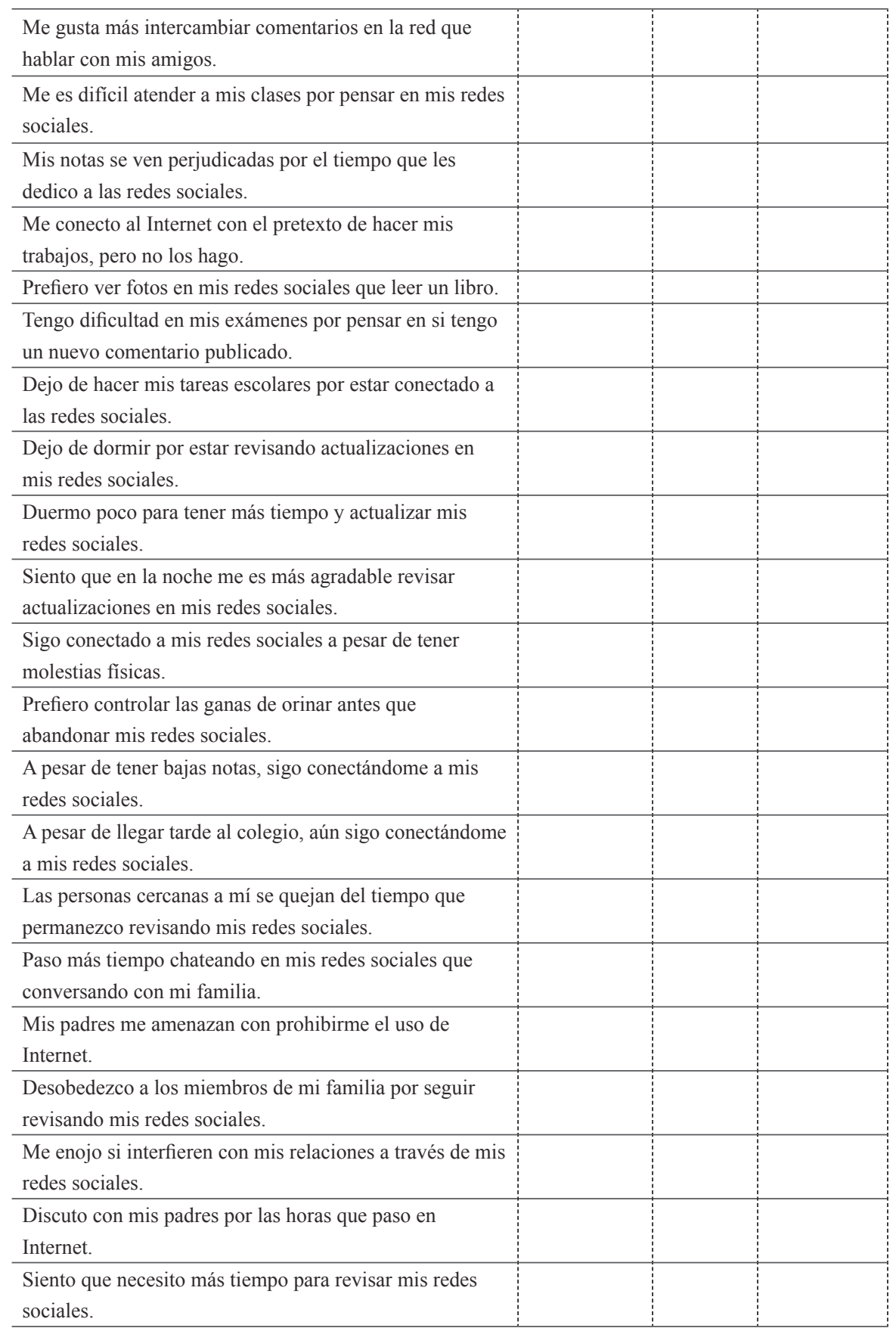




\section{Anexo B}

Lista de Chequeo de Habilidades Sociales (LCHS).

\section{INSTRUCCIONES}

A continuación encontrarás, enumerada, una lista de habilidades que las personas usan en su interacción social más o menos eficiente. Deberás determinar cómo usas cada una de esas habilidades, marcando con un aspa (x) en la columna de la derecha y en la fila correspondiente, según el siguiente patrón:

Marque en la columna:

NSi Nunca usas esa habilidad

RV Si Rara vez usas esa habilidad

AV Si A veces usas esa habilidad

AM Si A menudo usas esa habilidad

S Si Siempre usas esa habilidad

Trabaja rápidamente y no emplees mucho tiempo en cada pregunta; queremos tu primera reacción, no un proceso de pensamiento prolongado. Asegúrate de no omitir alguna pregunta. No hay respuesta "correcta" ni respuesta "incorrecta". Ahora comienza...

\begin{tabular}{|c|c|c|c|c|c|c|}
\hline & & $\mathbf{N}$ & RV & AV & $\mathbf{A M}$ & $\mathbf{S}$ \\
\hline 1. & $\begin{array}{l}\text { Escuchar: ¿Prestas atención a las personas que te están } \\
\text { hablando y haces un esfuerzo para comprender lo que están } \\
\text { diciendo? }\end{array}$ & & & & & \\
\hline 2. & $\begin{array}{l}\text { Iniciar una conversación: ¿Inicias conversaciones con otras } \\
\text { personas y luego las mantienes por un momento? }\end{array}$ & & & & & \\
\hline 3. & $\begin{array}{l}\text { Mantener una conversación: ¿Hablas con otras personas } \\
\text { sobre cosas de interés mutuo? }\end{array}$ & & & & & \\
\hline
\end{tabular}


4. Formular una pregunta: ¿Determinas la información que necesitas saber y se la pides a la persona adecuada?

5. Dar las gracias: ¿Permites que los demás sepan que estás agradecido con ellos por algo que hicieron contigo?

6. Presentarse: $¿$ Te esfuerzas por conocer personas por propia iniciativa?

7. Presentar a otras personas: ¿Ayudas a presentar a nuevas personas con otras?

8. Hacer un cumplido: ¿Dices a los demás lo que les gusta de ellos o de lo que hacen?

9. Pedir ayuda: ¿Pides ayuda cuando la necesitas?

10. Participar: ¿Eliges la mejor manera de acercarte a un grupo que está realizando una actividad y luego te integras a él?

11. Dar instrucciones: ¿Explicas instrucciones de tal manera que las personas puedan seguirlas fácilmente?

12. Seguir instrucciones: ¿Prestas cuidadosa atención a las instrucciones y luego las sigues?

13. Disculparte: ¿Pides disculpas a los demás cuando hacen algo que sabes que está mal?

14. Convencer a los demás: ¿Intentas persuadir a los demás de que tus ideas son mejores o más útiles que las de ellos?

15. Conocer tus propios sentimientos: ¿Intentas comprender y reconocer las emociones que experimentas?

16. Expresar tus sentimientos: ¿Permites que los demás conozcan lo que sientes?

17. Comprendes los sentimientos de los demás: ¿Intentas comprender lo que los demás sienten?

18. Enfrentarte con el enfado del otro: ¿Intentas comprender el enfado de la otra persona?

19. Expresar afecto: ¿Permites que los demás sepan que tú te interesas o te preocupas por ellos?

20. Resolver el miedo: ¿Cuándo sientes miedo, piensas por qué lo sientes y luego intentas hacer algo para disminuirlo?

21. Autor recompensarse: $¿$ Te das a ti mismo una recompensa después de que haces algo bien?

Pedir permiso: $¿$ Reconoces cuando es necesario pedir

22. permiso para hacer algo y luego lo pides a la persona indicada?

23.

Compartir algo: ¿Ofreces compartir tus cosas con los demás?

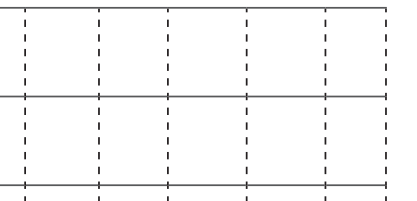

$\begin{array}{llllll}1 & 1 & 1 & 1 & 1 & 1 \\ 1 & 1 & 1 & 1 & 1 & 1 \\ 1 & 1 & 1 & 1 & 1 & 1 \\ 1 & 1 & 1 & 1 & 1 & 1 \\ 1 & 1 & 1 & 1 & 1 & 1 \\ 1 & 1 & 1 & 1 & 1 & 1 \\ 1 & 1 & 1 & 1 & 1 & 1 \\ 1 & 1 & 1 & 1 & 1\end{array}$

(1)

$\begin{array}{llllll}1 & 1 & 1 & 1 & 1 & 1 \\ 1 & 1 & 1 & 1 & 1 & 1 \\ 1 & 1 & 1 & 1 & 1 & 1 \\ 1 & 1 & 1 & 1 & 1\end{array}$

(




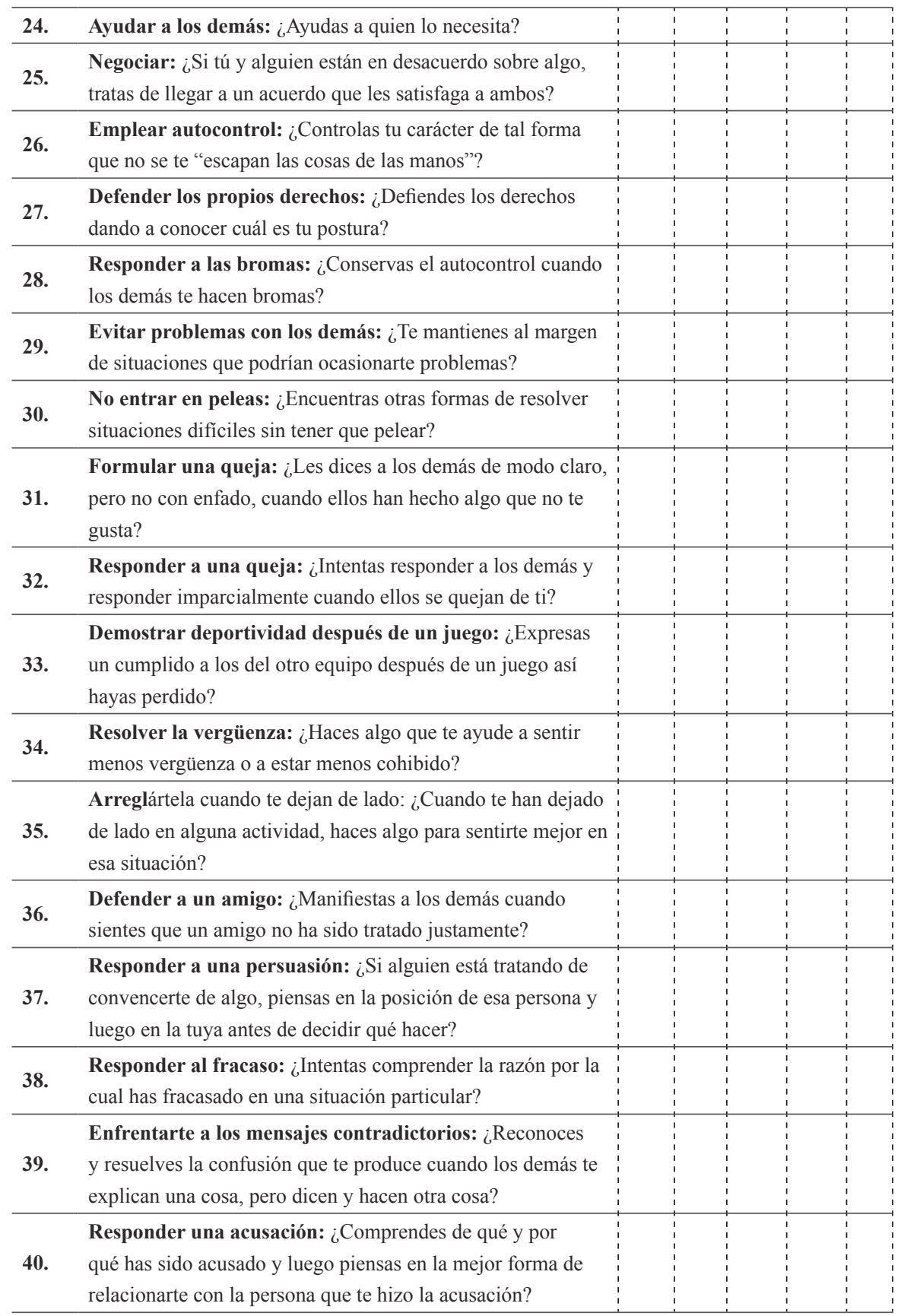


Prepararte para una conversación difícil: ¿Planificas la

41. mejor forma para exponer tu punto de vista antes de una conversación problemática?

Hacer frente a las presiones de grupo: ¿Decides qué cosas

42. quieres hacer cuando los demás quieren que hagas otra cosa distinta?

43. Tomar iniciativas: ¿Si te sientes aburrido, intentas encontrar

43. algo interesante de hacer?

44. Determinar la causa de un problema: $i$ Si surge un problema, intentas determinar qué lo causó?

45. Establecer un objetivo: ¿Determinas de manera realista lo que te gustaría realizar antes de empezar una tarea?

Determinar tus habilidades: ¿Determinas de manera

46. realista qué tan bien podrías realizar una tarea específica antes de iniciarla?

47. Reunir información: ¿Determinas lo que necesitas saber y cómo conseguir esa información?

\section{Resolver los problemas según su importancia:}

48. ¿Determinas de forma realista cuál de tus problemas es el más importante y el que debería de ser solucionado primero?

49. Tomar una decisión: ¿Consideras diferentes posibilidades y luego eliges la que te hará sentirte mejor?

50.

Concentrarte en una tarea: $¿$ Eres capaz de ignorar distracciones y sólo prestas atención a lo que quieres hacer? 